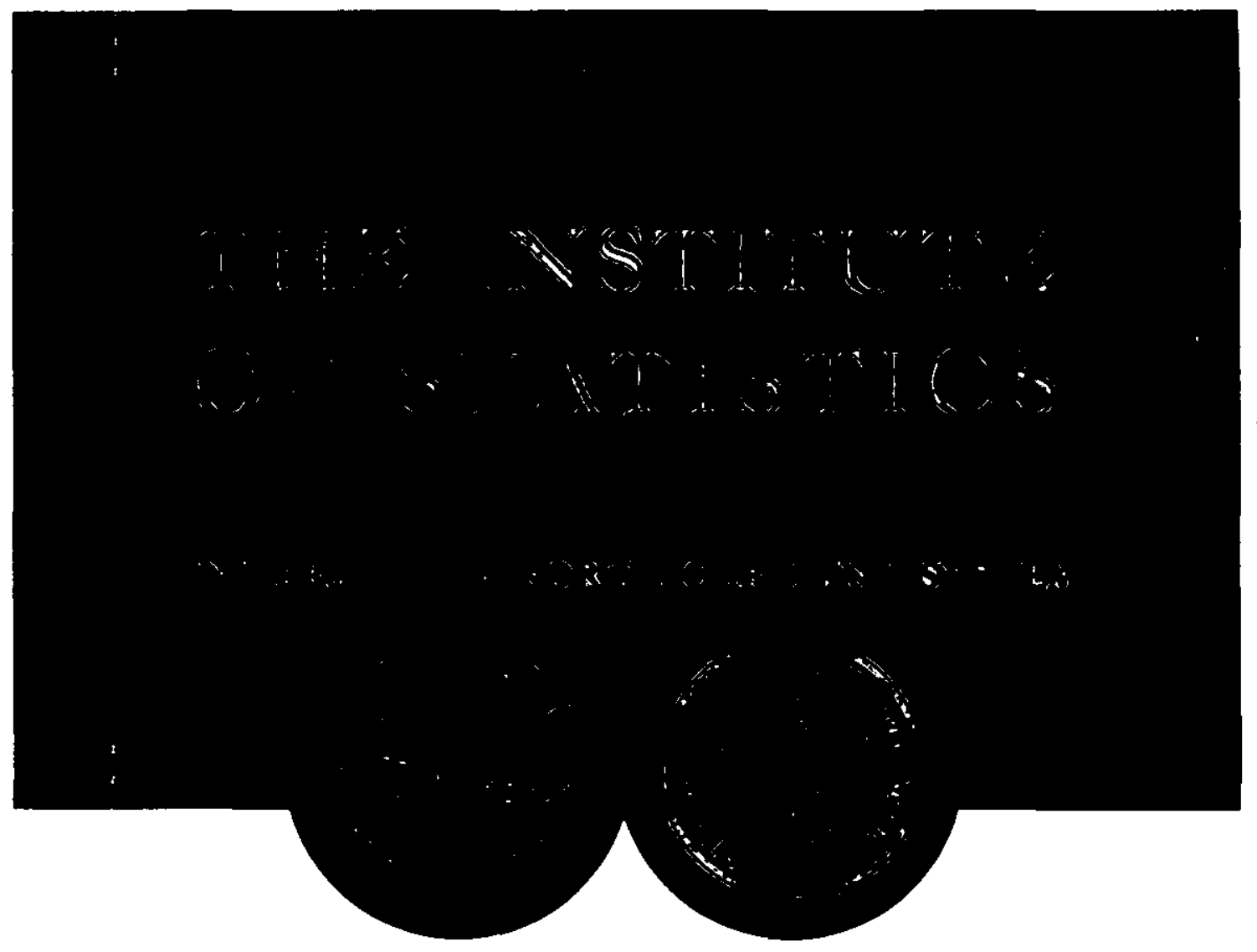

SIMULATION-EXTRAPOLATION ESTIMATION

IN PARAMETRIC MEASUREMENT ERROR MODELS

by

J. R. Cook and L. A. Stefanski

Institute of Statistics Mimeograph Series No. 2224R

Revised November 1993

NORTH CAROLINA STATE UNIVERSITY

Raleigh, North Carolina 


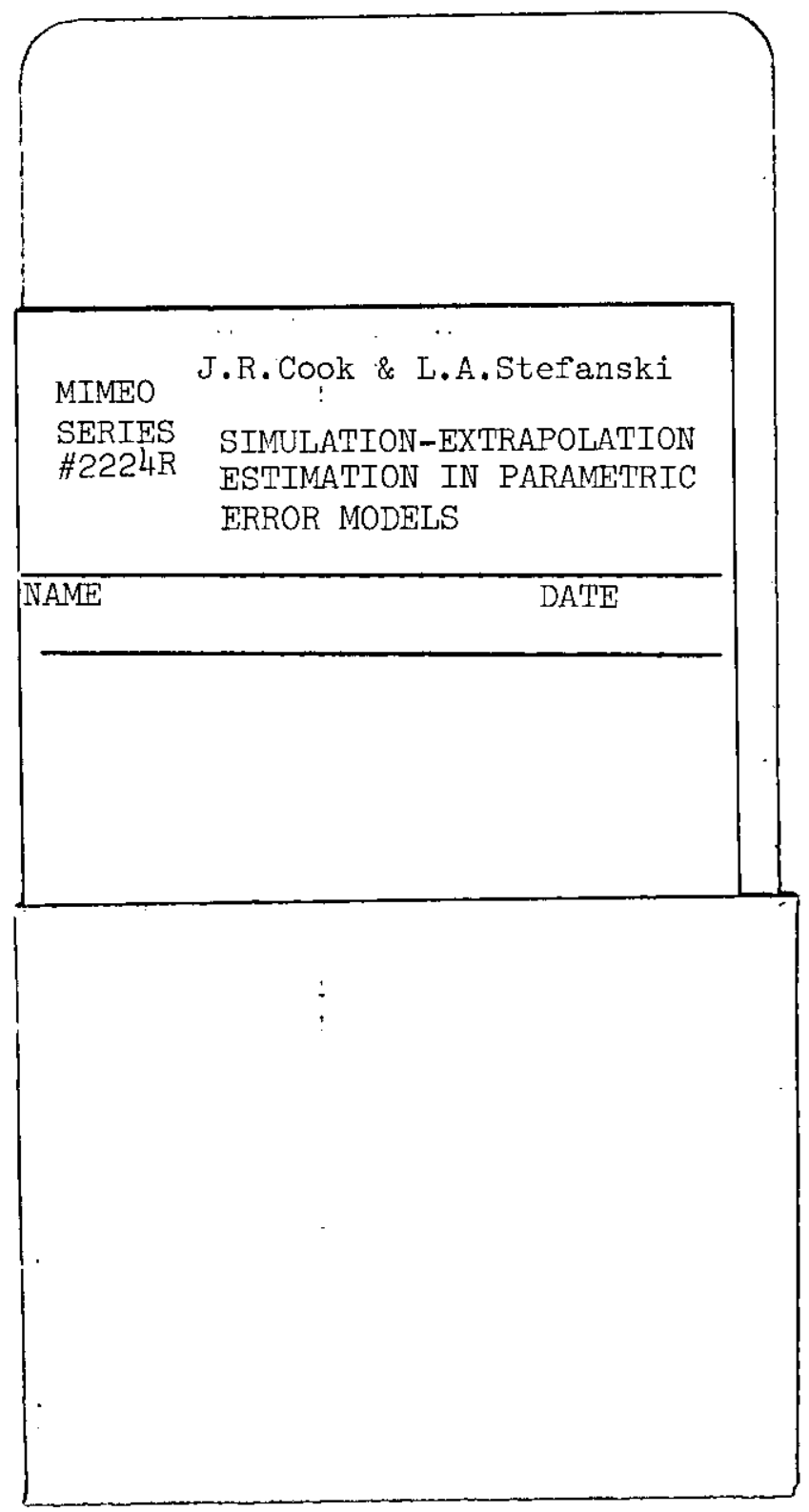




\title{
SIMULATION-EXTRAPOLATION ESTIMATION
}

\section{IN}

\section{PARAMETRIC MEASUREMENT ERROR MODELS}

\section{J. R. Cook}

Merck Research Laboratories

Division of Merck \& Co., Inc.

West Point, PA 19422

\author{
L. A. Stefanski \\ Department of Statistics \\ North Carolina State University \\ Raleigh, NC 27695
}

\begin{abstract}
We describe a simulation-based method of inference for parametric measurement error models in which the measurement error variance is known or at least well estimated. The method entails adding additional measurement error in known increments to the data, computing estimates from the contaminated data, establishing a trend between these estimates and the variance of the added errors, and extrapolating this trend back to the case of no measurement error.

We show that the method is equivalent or asymptotically equivalent to method-of-moments estimation in linear measurement error modelling. Simulation studies are presented showing that the method produces estimators that are nearly asymptotically unbiased and efficient in standard and nonstandard logistic regression models. An oversimplified but fairly accurate description of the method is that it is method-of-moments estimation using Monte Carlo derived estimating equations.
\end{abstract}

Note: This paper uses data supplied by the National Heart, Lung, and Blood Institute, NIH, and DHHS from the Framingham Heart Study. The views expressed in this paper are those of the authors and do not necessarily reflect the views of the National Heart, Lung, and Blood Institute, or of the Framingham Study. 


\section{INTRODUCTION}

In this paper we introduce a simulation-based method of inference for measurement error models applicable when the measurement error variance is known or can be reasonably well estimated, say from replicate measurements. It combines features of parametric-bootstrap and method-ofmoments inference. Estimates are obtained by adding additional measurement error to the data in a resampling stage, establishing a trend of measurement-error-induced bias versus the variance of the added measurement error, and extrapolating this trend back to the case of no measurement error.

The method lends itself to graphic description of both the effects of measurement error on parameter estimates and the nature of the ensuing "corrections for attenuation." It is also very simple to implement. This is particularly useful in applications where the user, though very familiar with standard statistical methods, may not be comfortable with the burdensome technical details of model fitting and statistical theory that generally accompanies all but the simplest of measurement error models. Since the method is completely general, it is also useful in applications when the particular model under consideration is novel and conventional approaches to estimation with the model have not been thoroughly developed/studied.

We developed the procedure in response to the need for fitting nonstandard generalized linear measurement error models, specifically models in which the mean function depends on something other than a linear function of the predictor measured with error. In principle, the general approaches described by Stefanski (1985), Fuller (1987, Ch. 3), Whittemore and Keller (1988), and Carroll and Stefanski (1990) could be applied to such models. However, in addition to their complexity and the need for specialized software, these methods entail approximations, the quality of which would be difficult to verify with complicated models.

Although the attenuating effects of measurement error have been well publicized and documented of late, see for example Palca (1990a,b) and MacMahon et al. (1990), it often happens that preliminary to a measurement error model analysis it is necessary to explain the need for such an analysis. The fact that measurement error in the predictor variable induces bias in least squares regression estimates is counter-intuitive to many, even those with training in statistics. 
A by-product of our method is a self-contained and highly relevant simulation study that clearly demonstrates the effect of measurement error and the need for bias correction(s).

When corrections for attenuation are made, they are often regarded by many with skepticism above and beyond the normal amount that should accompany any statistical modelling. The reason is that a correction for attenuation is often in the researcher's best interest. For example, assuming that the presence of an effect has been convincingly demonstrated, the perceived importance of the effect is likely to depend on its magnitude. A correction for attenuation generally increases the magnitude of the effect, thus it is in the researcher's interests to make such corrections. For an example in health economics, consider a situation wherein a pharmaceutical company is estimating the cost effectiveness of a blood pressure drug. A correction for attenuation due to measurement error in blood pressure readings produces a greater predicted decrease in risk for a given reduction in blood pressure, see MacMahon et al. (1990) and Palca (1990a,b). Clearly a correction for attenuation is in the company's best interest.

In the situations just described we believe that there is a need for corrections for attenuation that are both statistically and scientifically defensible while also being demonstratively conservative. By suitable adjustment of the extrapolation step, our method is capable of producing estimates with the desired properties. The trend line referred to in the first paragraph is generally monotone convex or concave and thus it is possible to obtain best linear-tangential approximations that result in conservative estimates.

\subsection{Organization of the Paper}

In this paper we describe our method in the context of parametric models with one predictor measured with error. The assumptions we employ are set forth in Section 2 along with a description of the basic method. A heuristic explanation of the procedure is given in this section as well.

An example illustrating the method is presented in Section 3. Section 4 describes the application of the method to standard and nonstandard linear and logistic regression models. Simulation results are used to demonstrate the utility of the procedure.

In Section 5 we consider application to a subset of data from the Framingham study. Here we use a novel resampling technique to illustrate the performance of the method with real data. We 
also consider fitting some nonstandard logistic regression models to illustrate the ease with which new and complicated models can be handled.

\section{BASIC METHOD and REFINEMENTS}

\subsection{Simulation Extrapolation Estimation}

The majority of applications involve regression models and we adopt a notation convenient for such problems. Let $Y, V, U$ and $X$ denote respectively the response variable, a covariate measured without error, the true predictor and the measured predictor. In this paper we restrict attention to cases in which $U$ and $X$ are scalars. Furthermore it is assumed that the observed data $\left\{Y_{i}, V_{i}, X_{i}\right\}_{1}^{n}$ are such that

$$
X_{i}=U_{i}+\sigma Z_{i}
$$

where $Z_{i}$ is a standard normal random variable independent of $U_{i}, V_{i}$ and $Y_{i}$, and $\sigma^{2}$ is the known measurement error variance.

We suppose the existence of an estimation procedure that maps a data set into the parameter space; for example, linear or logistic regression. Let $\theta \in \theta$ denote the parameter and $T$ the functional that maps the data set into $\Theta$. Then we can define the following estimators:

$$
\begin{gathered}
\hat{\theta}_{\mathrm{TRUE}}=T\left(\left\{Y_{i}, V_{i}, U_{i}\right\}_{1}^{n}\right) ; \\
\hat{\theta}_{\mathrm{NAIVE}}=T\left(\left\{Y_{i}, V_{i}, X_{i}\right\}_{1}^{n}\right) .
\end{gathered}
$$

Since $\hat{\theta}_{\text {TRUE }}$ depends on the unknown $\left\{U_{i}\right\}_{1}^{n}$, it is not an estimator in the strict sense. Nevertheless it is convenient to have a notation for this random vector.

For $\lambda \geq 0$, define

$$
X_{b, i}(\lambda)=X_{i}+\lambda^{1 / 2} \sigma Z_{b, i}
$$

where $\left\{Z_{b, i}\right\}_{i=1}^{n}$ are mutually independent, and independent of $\left\{Y_{i}, V_{i}, U_{i}, X_{i}\right\}_{1}^{n}$, and identically distributed standard normal random variables. Define

$$
\hat{\theta}_{b}(\lambda)=T\left(\left\{Y_{i}, V_{i}, X_{b, i}(\lambda)\right\}_{i=1}^{n}\right)
$$

and

$$
\hat{\theta}(\lambda)=E\left(\hat{\theta}_{b}(\lambda) \mid\left\{Y_{i}, V_{i}, X_{i}\right\}_{1}^{n}\right) ;
$$


that is, the expectation above is with respect to the distribution of $\left\{Z_{b, i}\right\}_{i=1}^{n}$ only.

Note that $\hat{\theta}(0)=\hat{\theta}_{b}(0)=\hat{\theta}_{\mathrm{NAIVE}}$. Exact determination of $\hat{\theta}(\lambda)$ for $\lambda>0$ is generally not feasible, but it can always be estimated arbitrarily well by generating a large number of independent measurement error vectors, $\left\{\left\{Z_{b, i}\right\}_{i=1}^{n}\right\}_{b=1}^{B}$, computing $\hat{\theta}_{b}(\lambda)$ for $b=1, \ldots, B$ and approximating $\hat{\theta}(\lambda)$ by the sample mean of $\left\{\hat{\theta}_{b}(\lambda)\right\}_{1}^{B}$. This is the simulation component of our method. We call the $\left\{Z_{b, i}\right\}_{i=1}^{n}$ pseudo errors.

The extrapolation step of the proposal entails modelling $\hat{\theta}(\lambda)$ as a function of $\lambda$ for $\lambda \geq 0$ and using the model to extrapolate back to $\lambda=-1$. This yields the simulation-extrapolation estimator denoted $\hat{\theta}_{\text {SIMEX }}$.

As an illustration of the SIMEX algorithm we describe its application to the familiar componentsof-variance model. With this model only $\left\{X_{i}\right\}_{1}^{n}$ is observed and $\theta=\sigma_{U}^{2}$, the variance of $U$. In this case $\hat{\theta}_{\mathrm{NAIVE}}=s_{X}^{2}$, the sample variance of $\left\{X_{i}\right\}_{1}^{n}$, and

$$
\begin{aligned}
\hat{\theta}_{b}(\lambda) & =\frac{1}{n-1} \sum_{i=1}^{n}\left(X_{b, i}(\lambda)-\bar{X}_{b}(\lambda)\right)^{2} \\
& =\frac{1}{n-1} \sum_{i=1}^{n}\left(X_{i}+\lambda^{1 / 2} \sigma Z_{b, i}-\bar{X}-\lambda^{1 / 2} \sigma \bar{Z}_{b}\right)^{2} .
\end{aligned}
$$

For this model it is well known that the expectation indicated in (2.1) is just

$$
\hat{\theta}(\lambda)=E\left(\hat{\theta}_{b}(\lambda) \mid\left\{X_{i}\right\}_{1}^{n}\right)=s_{X}^{2}+\lambda \sigma^{2}
$$

but we emphasize that it can also be estimated arbitrarily well by $B^{-1} \sum_{b=1}^{B} \hat{\theta}_{b}(\lambda)$.

In this case $\hat{\theta}(\lambda)$ is exactly linear in $\lambda \geq 0$ and extrapolation to $\lambda=-1$ results in $\hat{\theta}_{\text {SIMEX }}=$ $s_{X}^{2}-\sigma^{2}$, the familiar method-of-moments estimator. Note that $\hat{\theta}_{\text {SIMEX }}$ is an unbiased and consistent estimator of $\theta$.

\subsection{Heuristics}

In this section we give a heuristic explanation for SIMEX estimation. In later sections extensive simulation evidence for the procedure's utility in practice is presented.

Consider the naive estimator as the sample size $n$ increases. It converges in probability to some limit depending on the true value of the parameter, say $\theta_{0}$, and the measurement error variance $\sigma^{2}$. Call the limit $T\left(\theta_{0}, \sigma^{2}\right)$. If we assume that the naive estimator is a consistent estimator of $\theta_{0}$ in the absence of measurement error $\left(\sigma^{2}=0\right)$, then $T\left(\theta_{0}, 0\right)=\theta_{0}$. 
Now as $n$ increases $\hat{\theta}(\lambda)$ also converges in probability to some limit and we would expect that this limit can be represented as $T\left(\theta_{0}, \sigma^{2}+\lambda \sigma^{2}\right)$, at least under sufficient regularity conditions. Evaluating the latter expression at $\lambda=-1$, yields $T\left(\theta_{0}, \sigma^{2}-\sigma^{2}\right)=T\left(\theta_{0}, 0\right)=\theta_{0}$. Since $\hat{\theta}_{\text {SIMEX }}$ approximately estimates $T\left(\theta_{0}, \sigma^{2}+\lambda \sigma^{2}\right)_{\lambda=-1}$, it will be approximately consistent for $\theta_{0}$ in general.

\subsection{Some Refinements}

There may be estimators for which the expectation indicated in (2.1) does not exist. This may or may not cause problems with the use of the sample mean as a location estimator in the simulation step. However, it may be necessary to summarize the simulated estimates with a robust estimator of location such as the median.

Our measurement error model assumes independence between measurement errors and the other variables in the data sets. Although the IID pseudo errors are generated under these assumptions, the simulated sets of errors have nonzero sample correlations with $Y, V$ or $X$, nonzero sample means, and sample variances $\neq 1$. The effects of these random departures from expected behavior can be eliminated by generating the pseudo errors to be uncorrelated with the observed data and normalized to have sample mean and variance equal to 0 and 1 respectively. We call these NONIID pseudo errors. Using NON-IID pseudo errors improves the convergence of $B^{-1} \sum_{b=1}^{B} \hat{\theta}_{b}(\lambda)$ to $\hat{\theta}(\lambda)$ as $B \rightarrow \infty$. For example, if NON-IID pseudo errors are used in the components-of-variance application, $B^{-1} \sum_{b=1}^{B} \hat{\theta}_{b}(\lambda)=\hat{\theta}(\lambda)$ for all $B$.

\subsection{Methods of Extrapolation}

The Achilles' heel of our proposal is the extrapolation step. If this cannot be done in a convincing and demonstrably useful fashion, our strategy is of limited utility. However, even when the extrapolation step is unreliable, the plot of $\hat{\theta}(\lambda)$ versus $\lambda$ is still informative, especially in complex models where the direction and approximate magnitude of the measurement-error induced bias is not obvious. Note that in the components-of-variance model the extrapolation was exact.

In the simulation results that follow we employed three extrapolants: (i) a two-point linear extrapolation; (ii) a three-point quadratic extrapolation; and (iii) a nonlinear extrapolation based on fitting mean models of the form $\mu(\lambda)=a+b(c+\lambda)^{-1}$ to the components in $\hat{\theta}(\lambda)$. 


\section{An Example}

We now present an example to describe the type of analysis we have in mind and to point out some concessions for computational speed that were made in the simulations that follow.

We generated $n=1500$ independent observations from the logistic regression measurement error model

$$
\begin{gathered}
X=U+\sigma Z, \\
\operatorname{pr}(Y=1 \mid U, V)=F\left(\beta_{1}+\beta_{U} U+\beta_{V} V+\beta_{U V} U V\right)
\end{gathered}
$$

where $F$ is the logistic distribution function and $(U, V)$ are bivariate normal with zero means, unit variances and correlation $=1 / \sqrt{5} \approx 0.447$. The regression parameters were set at $\beta_{1}=-2$, $\beta_{U}=1, \beta_{V}=0.25$ and $\beta_{U V}=0.25$, and we set $\sigma^{2}=0.5$.

The sample size is consistent with large epidemiologic studies of the type motivating much of the current research in measurement error models, although the parameter values were not matched with any particular study. A full simulation study of this model is described in Section 4.5. Here we analyze one generated data set.

Our algorithm calls for generating pseudo errors $\left\{Z_{b, i}\right\}_{i=1}^{n}$, forming pseudo predictors $X_{b, i}(\lambda)=$ $X_{i}+\lambda \sigma Z_{b, i}$, and fitting the logistic model (3.1) to $\left\{Y_{i}, V_{i}, X_{b, i}(\lambda)\right\}_{i=1}^{n}$ for several values of $\lambda$. This is repeated for $b=1, \ldots, B$.

In any particular application, the grid of lambda values can be extensive and $B$ can be chosen so large that the Monte Carlo error is negligible, without making the computations prohibitively time consuming. Computing time is a factor in simulation studies, however. Thus in the simulation studies that follow we employed a relatively course grid, $\lambda \in\{0,0.5,1.0,1.5,2.0\}$, and $B$ was chosen after some preliminary investigations of the Monte Carlo variability and computational time. However, for this example we took $\lambda \in\{0,1 / 8, \ldots, 15 / 8,16 / 8\}$, with $B=50$.

In all our work only one set of pseudo errors was used to calculate the estimates $\hat{\theta}_{b}\left(\lambda_{j}\right)$ for different values of $\lambda_{j}$ for a fixed $b$. That is, for a given $b, \hat{\theta}_{b}\left(\lambda_{j}\right)$ was calculated from $\left\{Y_{i}, V_{i}, X_{i}+\lambda_{j}^{1 / 2} \sigma Z_{b, i}\right\}_{i=1}^{n}$, where $Z_{b, i}$ did not vary with $j$.

Figures 1a-d display the results of the Monte Carlo step and the extrapolation step. Although the $\hat{\theta}(\lambda)$ are plotted for the the large grid defined above, only the four points $\lambda=.5,1,1.5,2.0$ 

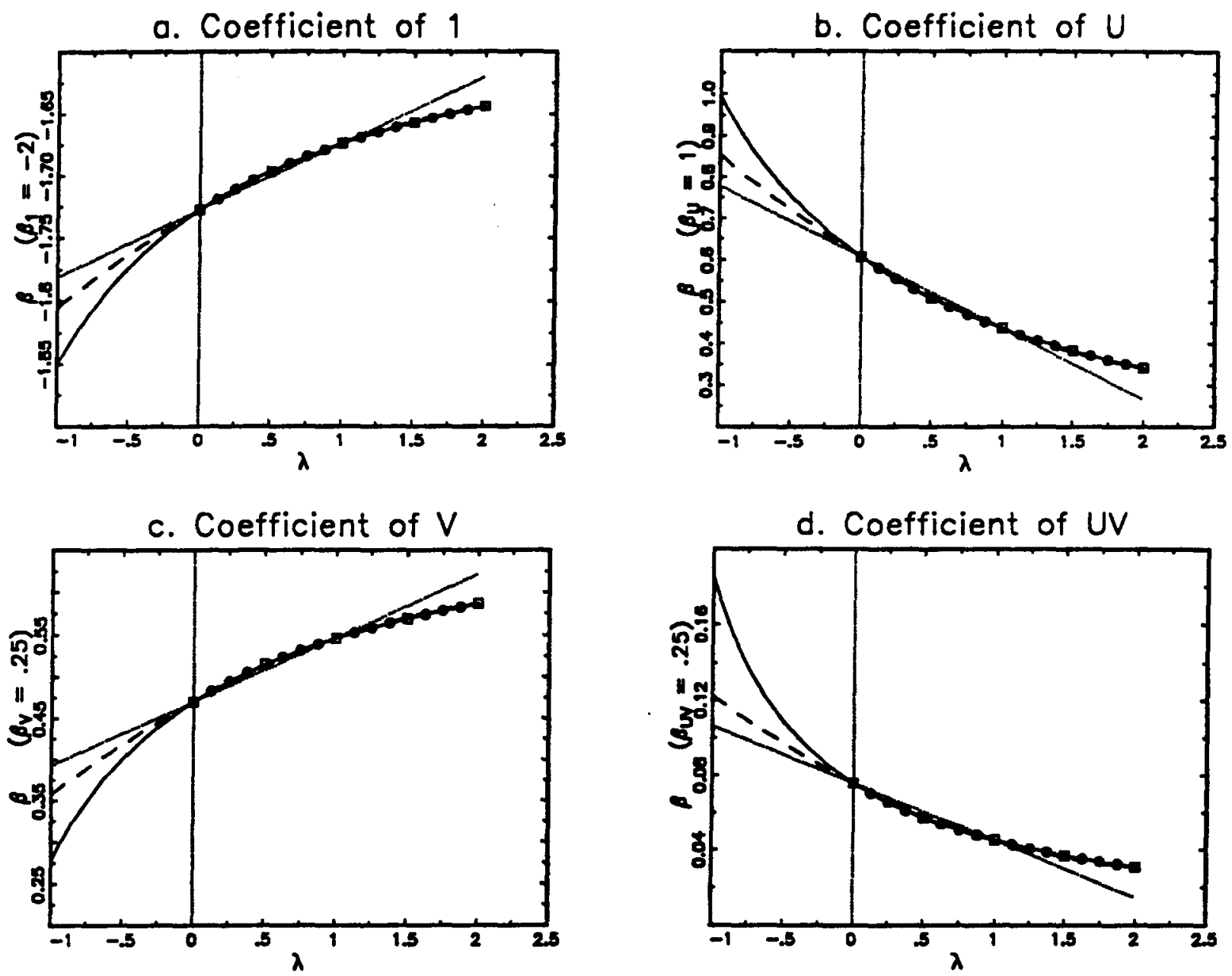

Figures 1a-d. Plots of $\hat{\theta}(\lambda)$ versus $\lambda$ for the model (2.2) of Section 3. Linear, quadratic and nonlinear extrapolants indicated by dotted, dashed and solid lines respectively. 
(open boxes), and the naive estimate $\lambda=0$ (open box), were used in the extrapolation step. Linear extrapolations used $\lambda=0,1$; quadratic extrapolations used $\lambda=0,1,2$; and the nonlinear extrapolations used the five points plotted with open boxes to fit the model.

The linear, quadratic and nonlinear fits and extrapolants are graphed with dotted, dashed and solid curves respectively. These graphs were made from the NON-IID pseudo errors with the sample mean as location estimator. The other combinations of location estimator and IID/NON-IID errors produced nearly identical results for this data set.

The plots make the qualitative effect, i.e., the direction of the bias, of the measurement error readily apparent. Although it might be argued that the plot in $1 \mathrm{~b}$ merely confirms intuition, the same cannot be said of the plots in $1 \mathrm{a}, \mathrm{c}$ and $\mathrm{d}$.

For this data set and choice of $\left\{\lambda_{j}\right\}$, the estimators are well ordered with ordering NAIVE > LINEAR > QUADRATIC > NONLINEAR, with respect to estimation error, i.e. the NAIVE estimate is worst, the NONLINEAR extrapolant is best for all regression coefficients.

The fit of the nonlinear model is excellent and instills confidence in the extrapolation step. Remember that only the box points were used in fitting the model. In later sections we prove that the mean function $a+b(c+\lambda)^{-1}$ is exactly appropriate for linear models, but we were unexpectedly surprised by the quality of the fit in logistic models.

The plots suggest that a two-point extrapolation based on a small positive value of $\lambda$ and $\lambda=0$ would perform well. Our experience to date suggests that this is the case. The plots of $\hat{\theta}(\lambda)$ are generally convex or concave and thus a tangential approximation is the best linear extrapolant in a conservative sense. The problem is that a two-point extrapolation based on two close $\lambda s$ is very sensitive to the Monte Carlo sampling error. Thus we have not studied it in our simulations. However, it should perform well whenever Monte Carlo error can be made negligible.

Also it is likely that a quadratic extrapolant based on three $\lambda$ s close to zero would work well provided the Monte Carlo error is negligible. Our experience is that the curvature in the graph of $\hat{\theta}(\lambda)$ increases as $\lambda$ decreases. In such cases it is possible to argue that a local three-point quadratic extrapolation would be conservative in the same sense as a tangential linear extrapolant.

Figure 2 illustrates the estimated response functions corresponding to the SIMEX estimators 


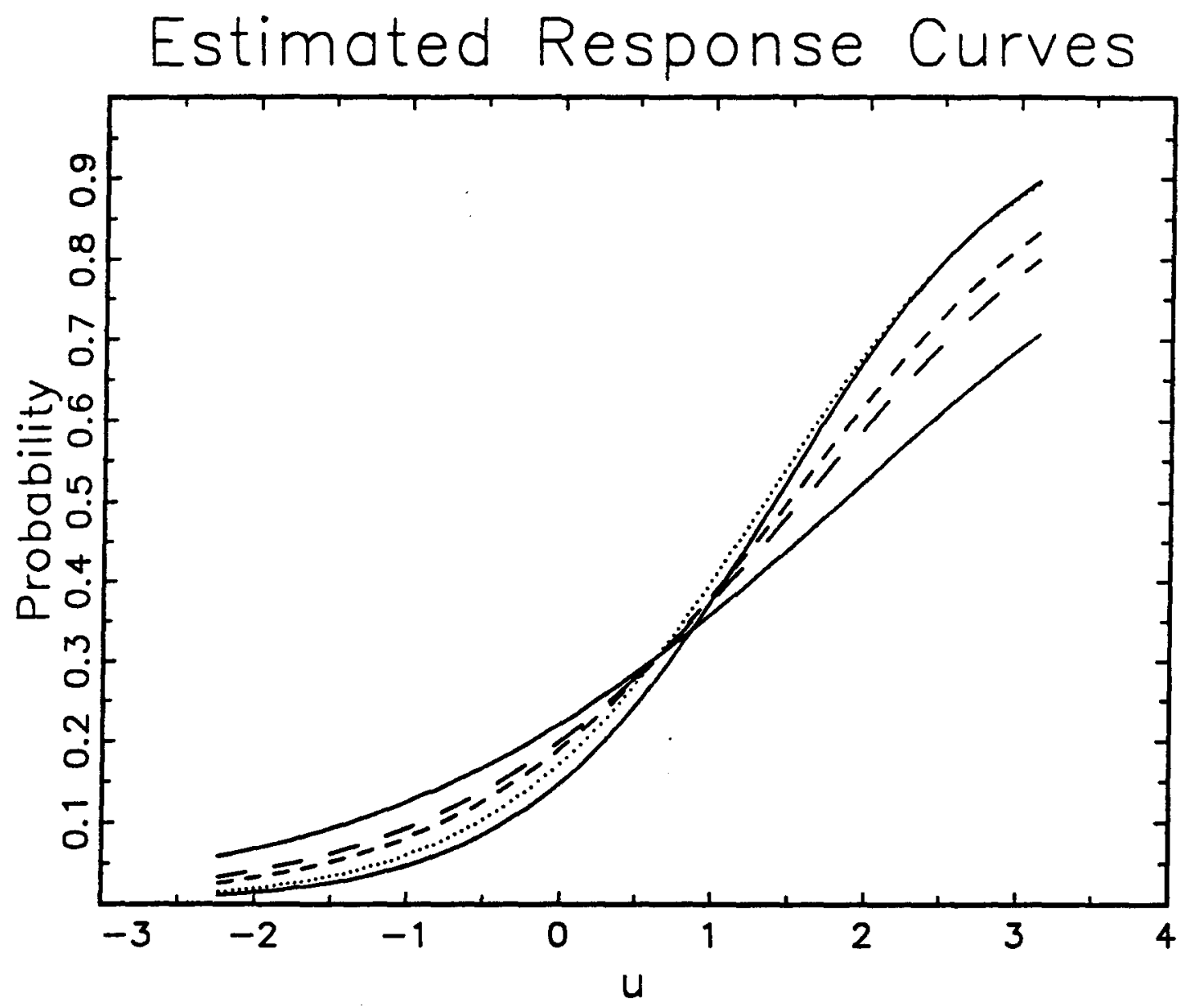

Figure 2. Estimated Response Functions for Example in $\oint 3$. Solid, NAIVE and estimand; long dashes, LINEAR; short dashes, QUADRATIC; dotted, NONLINEAR. 
and the NAIVE estimator. Graphs of (3.1) are plotted with $v=1$ and $-5 / \sqrt{5} \leq u \leq 7 / \sqrt{5}$. The solid curve with the least average slope corresponds to the NAIVE estimate. The other solid curve is the population response curve, i.e., the estimand. The long-dashed, short-dashed and dotted curves correspond to the linear, quadratic and nonlinear extrapolants respectively. Note that the conservatism of the linear and quadratic extrapolants manifests itself in the estimated response functions as well.

\section{Application to Parametric Models}

\subsection{Simple Linear Regression}

We developed the simulation/extrapolation procedure primarily for use in models for which the exact effects of measurement error cannot be determined. However, it is instructive to investigate how new methods perform in familiar, well-understood models and hence we study the simple errors-in-variables model first. An added benefit of studying this model is that the validity of the heuristics in Section 2.1 is readily confirmed.

We consider least-squares estimation of a line. Thus the functional $T$ returns least-squares estimates of an intercept and slope. Let $\theta=\left(\beta_{1}, \beta_{U}\right)^{T}$. Then

$$
\hat{\beta}_{1}(\lambda)=E\left\{\bar{Y}-\hat{\beta}_{U}(\lambda)\left(\bar{X}+\lambda^{1 / 2} \sigma \bar{Z}_{b}\right) \mid\left\{Y_{i}, X_{i}\right\}_{1}^{n}\right\}
$$

and

$$
\hat{\beta}_{U}(\lambda)=E\left\{\frac{S_{Y X}+\lambda^{1 / 2} \sigma S_{Y Z_{b}}}{S_{X X}+\lambda \sigma^{2} S_{Z_{b} Z_{b}}+2 \lambda^{1 / 2} \sigma S_{X Z_{b}}} \mid\left\{Y_{i}, X_{i}\right\}_{1}^{n}\right\},
$$

where conventional notation is used for sums of centered cross products. For the NON-IID pseudo errors, $S_{Y Z_{b}}=S_{X Z_{b}}=\bar{Z}_{b}=0$ and $S_{Z_{b} Z_{b}}=n$, and thus

$$
\hat{\beta}_{1}(\lambda)=\bar{Y}-\hat{\beta}_{U}(\lambda) \bar{X} \quad \text { and } \quad \hat{\beta}_{U}(\lambda)=\frac{S_{Y X}}{S_{X X}+n \lambda \sigma^{2}} .
$$

Note that both $\hat{\beta}_{1}(\lambda)$ and $\hat{\beta}_{U}(\lambda)$ in (4.1) are functions of $\lambda$ of the form $a+b(c+\lambda)^{-1}$ and that evaluation at $\lambda=-1$ yields the usual method-of-moments estimators (Fuller 1987, p. 14). Asymptotically $(n \rightarrow \infty)$ the difference between estimators based on the NON-IID and IID pseudo errors is negligible. Thus (4.1) holds asymptotically for the IID pseudo errors as well.

Although the nonlinear extrapolant estimators derived from the NON-IID pseudo data are identical to the usual method-of-moments estimator, the other combinations of extrapolant type, 
location functional, and error type yield different SIMEX estimators. We conducted a simulation study to compare these estimators with some common errors-in-variables estimators. In addition to the SIMEX estimators we included in the study the method-of-moments estimator (MOM), Fuller's modified moment estimators (Fuller, 1987, p. 164) with $\alpha=2$ and 5 (F2 and F5), and two estimators proposed by Cook (1989) (C1 and C2). Cook (1989) studied estimators of the form

$$
\hat{\beta}_{U, k}=\frac{1-\hat{\delta}^{k+1}}{1-\hat{\delta}} \hat{\beta}_{\mathrm{NAIVE}}
$$

where $\hat{\delta}=n \sigma^{2} / S_{X X}$ and determined $k$ to minimize an approximation to the mean squared error of the estimator. The two estimators included in our study correspond to two estimates of the optimal $k$,

$$
\hat{k}_{1}=\frac{\log \left(\frac{-n(1-\hat{\delta}) \log \hat{\delta}}{2 \log n}\right)}{\log (1 / \hat{\delta})} \quad \text { and } \quad \hat{k}_{2}=\frac{\log n}{\log (1 / \hat{\delta})} .
$$

In our simulation we employed a structural measurement error model

$$
\left(\begin{array}{c}
Y_{i} \\
X_{i} \\
U_{i}
\end{array}\right) \text { iid } \mathrm{N}\left\{\left(\begin{array}{l}
0 \\
0 \\
0
\end{array}\right),\left(\begin{array}{lll}
2 & 1 & 1 \\
1 & 2 & 1 \\
1 & 1 & 1
\end{array}\right)\right\}, \quad i=1, \ldots, 100
$$

Four-hundred independent data sets were generated and analyzed. The SIMEX estimators were computed with $B=100$.

The results of the simulation study for the slope $\left(\beta_{U}=1\right)$ are displayed as kernel density estimates in Figure 3. A normal kernel was used and the same bandwidth was used for all of the density estimates. The common bandwidth was set to $.3 \times$ the median of the standard deviations of all the estimators in the study.

There are six well-defined groups among the estimators. Groups I, II and VI have only a single member each, $\hat{\beta}_{U, T R U E}, \hat{\beta}_{U, N A I V E}$ and $\hat{\beta}_{U, C 1}$ respectively. Groups III and IV have four members each, corresponding to the $2^{2}$ factorial of location estimator (mean, median) $\times$ error type (IID, NON-IID), for the linear and quadratic extrapolants respectively. Group V contains $\hat{\beta}_{U, M O M}$, $\hat{\beta}_{U, \mathrm{~F} 2}, \hat{\beta}_{U, \mathrm{~F} 5}, \hat{\beta}_{U, \mathrm{C} 2}$, and the two SIMEX estimators obtained by the nonlinear extrapolation of the IID pseudo data using the mean and median location functionals. It also contains the SIMEX estimators based on the NON-IID pseudo errors, since the latter are equivalent to the method-ofmoments estimator for simple linear regression. 


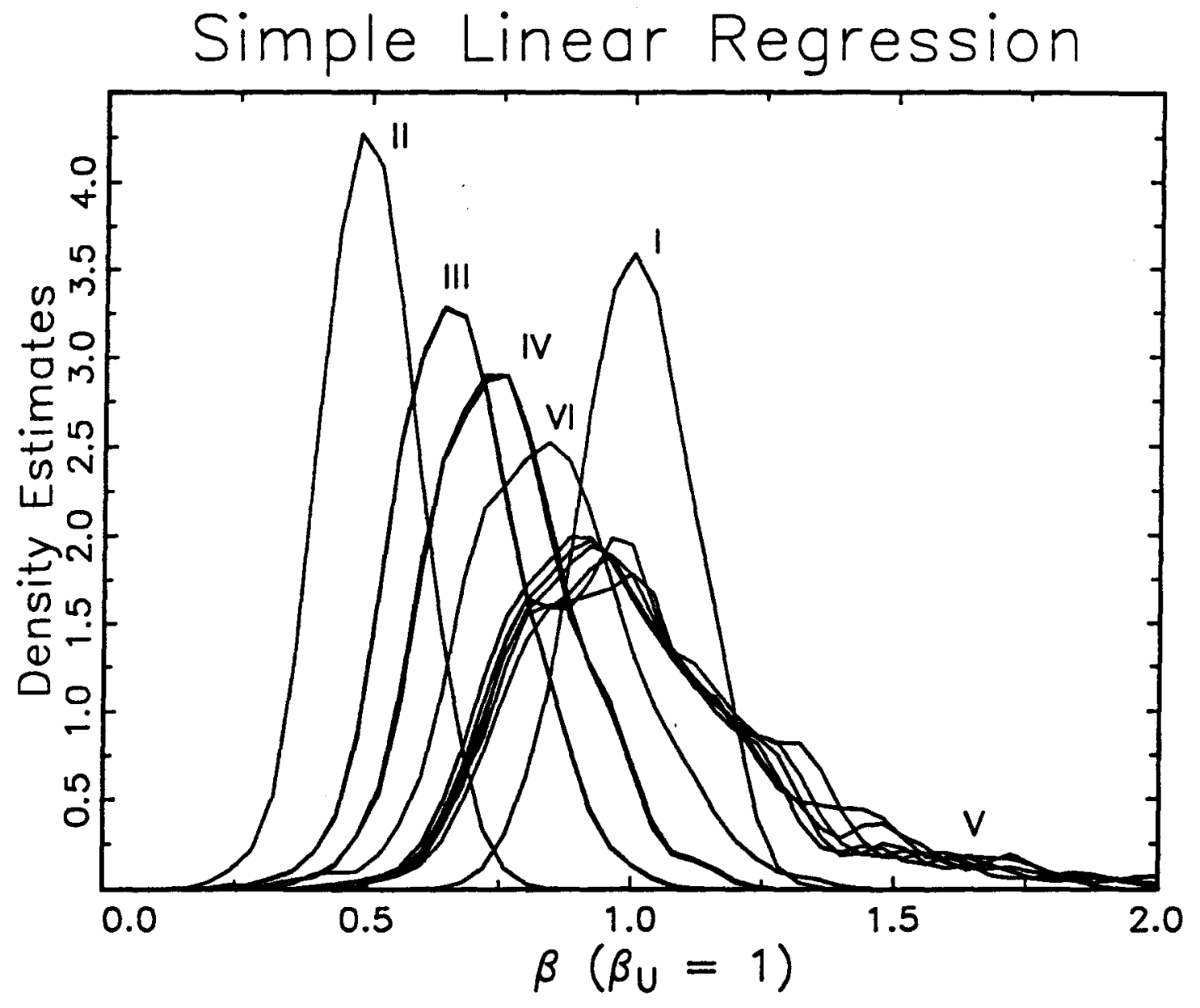

Figure 3. Kernel density estimates of the distributions of the slope $\left(\beta_{U}\right)$ estimators in the simple linear regression simulation. 
The simulation sample (400) is not large enough to allow detailed comparisons between estimators within groups. However, this was not our intent. Rather we wanted to demonstrate the conservative behavior of the linear and quadratic extrapolants and the method-of-moments-like performance of the nonlinear extrapolants.

\subsection{Multiple Linear Regression}

Instead of presenting simulation results for the multiple linear regression model, we will prove that the nonlinear SIMEX estimators based on NON-IID pseudo errors are identical to the usual method-of-moments estimators. We adopt a different notation in this section in order to make best use of standard linear algebra results. Let $U_{i}$ denote the true $p$-dimensional predictor with one component, the first, measured with error, and let $X_{i}$ denote the $p \times 1$ observed predictor. The vector of regression coefficients is $\beta$.

Then for the NON-IID pseudo data it is readily shown that

$$
\hat{\beta}(\lambda)=\left\{X^{T} X+\left(\begin{array}{cc}
\lambda \sigma^{2} & 0 \\
0 & 0
\end{array}\right)\right\}^{-1} X^{T} Y
$$

Clearly $\hat{\beta}(-1)$ is the common method-of-moments estimator (Fuller, 1987, p. 105). Now write

$$
X^{T} X=\left(\begin{array}{cc}
A & B^{T} \\
B & C
\end{array}\right)
$$

Then

$$
\left(\begin{array}{cc}
A+\lambda \sigma^{2} & B^{T} \\
B & C
\end{array}\right)\left(\begin{array}{c}
\hat{\beta}_{1}(\lambda) \\
\hat{\beta}_{2}(\lambda)
\end{array}\right)=\left(\begin{array}{l}
k_{1} \\
k_{2}
\end{array}\right)
$$

where $\left(\hat{\beta}_{1}(\lambda), \hat{\beta}_{2}(\lambda)^{T}\right)^{T}=\hat{\beta}(\lambda)$ and $\left(k_{1}, k_{2}^{T}\right)^{T}=X^{T} Y$ independent of $\lambda$. Solving this system yields

$$
\begin{aligned}
& \hat{\beta}_{1}(\lambda)=\frac{k_{1}-B^{T} C^{-1} k_{2}}{A-B^{T} C^{-1} B+\lambda \sigma^{2}} \\
& \hat{\beta}_{2}(\lambda)=C^{-1} k_{2}-\frac{C^{-1} B k_{1}-C^{-1} B B^{T} C^{-1} k_{2}}{A-B^{T} C^{-1} B+\lambda \sigma^{2}}
\end{aligned}
$$

showing that all of the components of $\hat{\beta}(\lambda)$ are functions of $\lambda$ of the form $a+b(c+\lambda)^{-1}$. 


\subsection{Multiple Linear Regression with Interaction}

Next we consider the measurement error model

$$
\begin{aligned}
\left(\begin{array}{l}
U_{i} \\
V_{i}
\end{array}\right) \text { iid } \mathrm{N} & \left\{\left(\begin{array}{l}
0 \\
0
\end{array}\right),\left(\begin{array}{ll}
1 & 0 \\
0 & 1
\end{array}\right)\right\}, \quad i=1, \ldots, 200, \\
X_{i} \mid U_{i} & \sim \mathrm{N}\left(U_{i}, 1\right), \\
Y_{i} \mid\left(U_{i}, V_{i}\right) & \sim \mathrm{N}\left(\beta_{1}+\beta_{U} U_{i}+\beta_{V} V_{i}+\beta_{U V} U_{i} V_{i}, 1\right),
\end{aligned}
$$

where $\beta_{1}=0, \beta_{U}=\beta_{V}=\beta_{U V}=1$.

The benchmark estimator for this model is the method-of-moments estimator

$$
\hat{\beta}_{\mathrm{MOM}}=\left(D^{T} D-C\right)^{-1} D^{T} Y
$$

where $\beta=\left(\beta_{1}, \beta_{U}, \beta_{V}, \beta_{U V}\right)^{T}, D$ is the $200 \times 4$ design matrix $(1, U, V, U V)$ and

$$
C=\left(\begin{array}{cccc}
0 & 0 & 0 & 0 \\
0 & 1 & 0 & \bar{V} \\
0 & 0 & 0 & 0 \\
0 & \bar{V} & 0 & \left(\bar{V}^{2}+s_{V}^{2}\right)
\end{array}\right) ;
$$

see Fuller (1984) and Hwang (1986).

Figures 4a-d display the results of a simulation study of this model. The SIMEX estimators were computed with $B=200$. Four hundred data sets were generated and analyzed. The kernel density estimates were calculated as in the simple linear regression simulation study.

The estimators again lie in well-defined groups. Groups I and II contain the TRUE and NAIVE estimators respectively. Groups III and IV contain the linear and quadratic extrapolant SIMEX estimators respectively. Group V includes the nonlinear extrapolant SIMEX estimators and the method-of-moments estimator.

The conservative behavior of the linear and quadratic extrapolants and the method-of-momentslike performance of the nonlinear extrapolants is readily apparent.

\subsection{Multiple Logistic Regression}

The model for the study is

$$
\begin{aligned}
&\left(\begin{array}{l}
U_{i} \\
V_{i}
\end{array}\right) \text { iid } \mathrm{N}\left\{\left(\begin{array}{l}
0 \\
0
\end{array}\right),\left(\begin{array}{ll}
1 & 0 \\
0 & 1
\end{array}\right)\right\}, \quad i=1, \ldots, 1500, \\
& X_{i} \mid U_{i} \sim \mathrm{N}\left(U_{i}, 1\right), \\
& \operatorname{Pr}\left(Y_{i}=1 \mid U_{i}, V_{i}\right) \sim F\left(\beta_{1}+\beta_{U} U_{i}+\beta_{V} V_{i}\right),
\end{aligned}
$$


a. Coefficient of 1

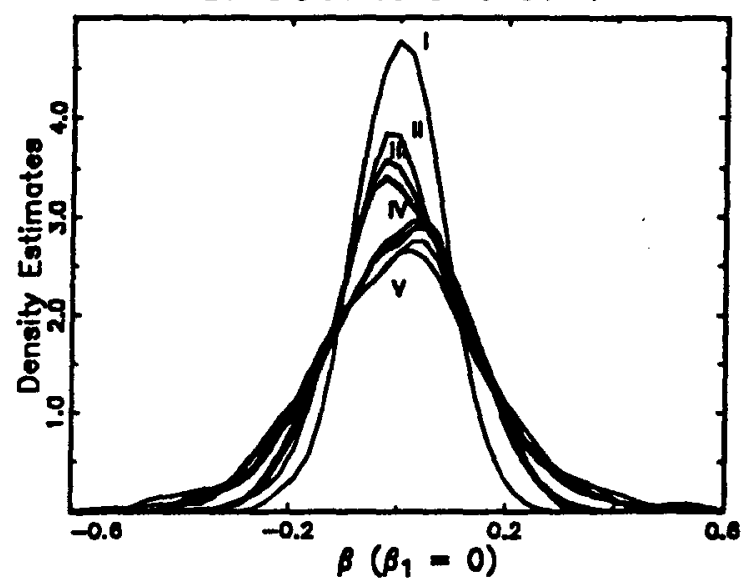

c. Coefficient of $V$

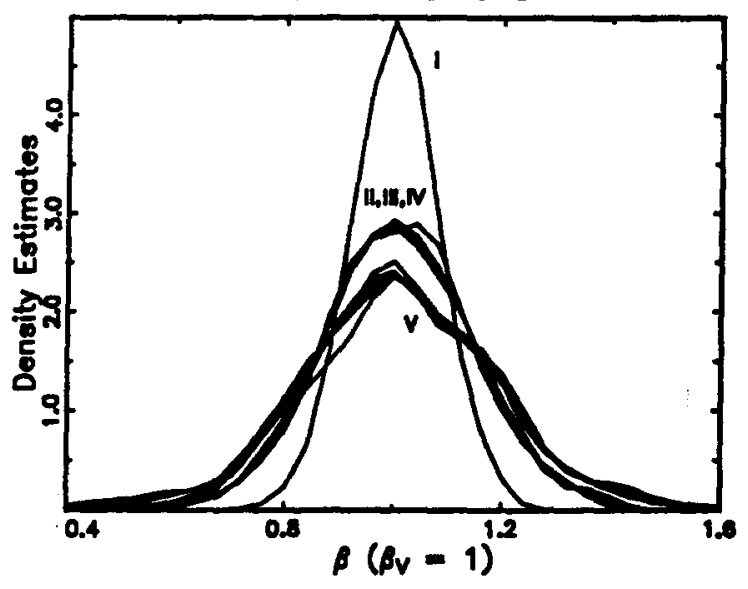

b. Coefficient of $U$

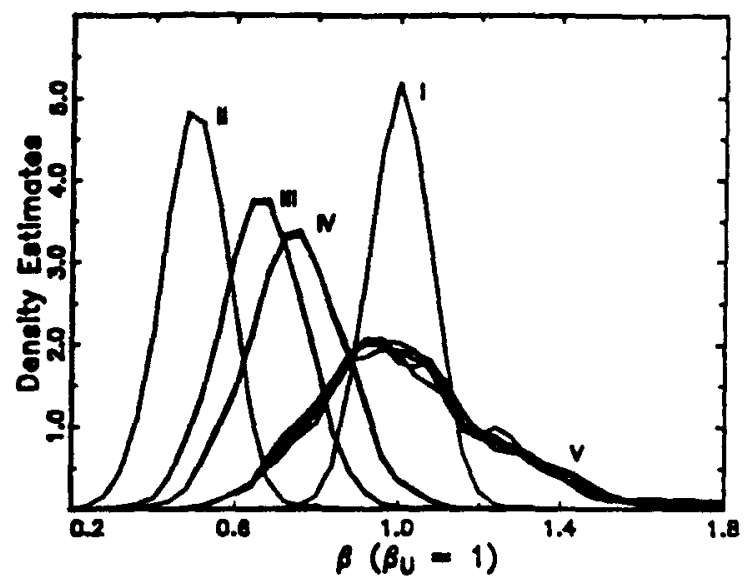

d. Coefficient of UV

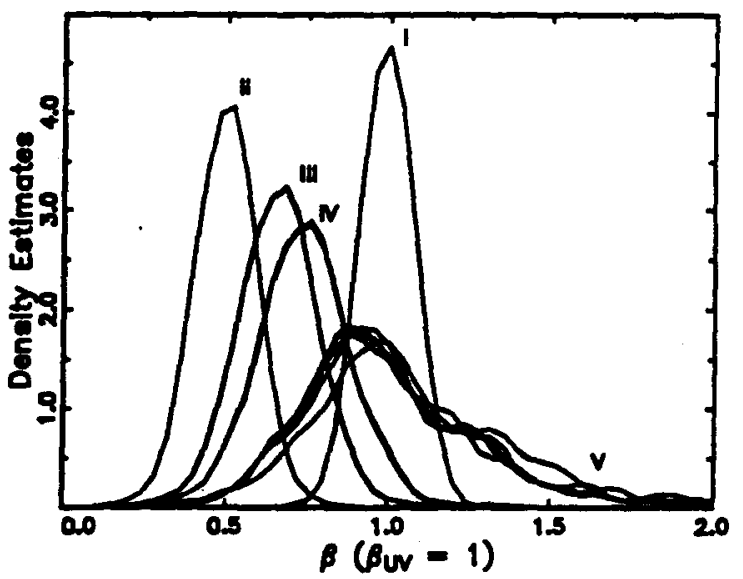

Figures 4a-d. Kernel density estimates of the distributions of the regression parameters in the multiple linear regression with interaction model simulation. 
where $\beta_{1}=-2, \beta_{U}=1, \beta_{V}=.5$.

There are a number of estimators that have been proposed for this model. Only the so-called sufficiency estimator of Stefanski and Carroll $(1985,1987)$ is known to be generally consistent and asymptotically efficient in the absence of parametric distributional assumptions on $U$ and thus we included only it in our Monte Carlo study. It provides a benchmark for comparison just as the methods-of-moments estimators did for the linear models simulations. The estimating equations for the sufficiency estimator and its optimality properties are discussed in the previously cited papers.

Figures 5a-c display the results of the simulation study of this model. The SIMEX estimators were computed with $B=50$. Four hundred data sets were generated and analyzed. The kernel density estimates were calculated as in the previous simulation studies.

Groups I and II correspond to the TRUE and NAIVE estimates respectively, Groups III and IV to the linear and quadratic extrapolant estimators, and Group V contains the nonlinear extrapolant SIMEX estimators and the sufficiency estimator. The groupings are well defined for the intercept and $\beta_{U}$, somewhat less so for $\beta_{V}$. Again we see the conservative behavior of the linear and nonlinear extrapolants. More interesting is the near indistinguishability between the distributions of the nonlinear extrapolants and the sufficiency estimator, the latter being asymptotically efficient among all estimators that do not assume a parametric form for the distribution of $U$.

Figures 5d-f display the results of a second simulation study of this model with the difference that the error in $X$ as a measurement of $U$ was generated as a standardized (mean 0 , variance 1 ) uniform random variable. The SIMEX procedure generates normal errors in the simulation step, thus the issue of robustness naturally arises. Although we have not studied this in detail, Figures $5 \mathrm{~d}-\mathrm{f}$ suggest that at least for the logistic model the SIMEX estimators behave similarly for normal and uniform measurement errors.

\subsection{Multiple Logistic Regression with Interaction}

We now present the results from a simulation study of the model (3.1) of Section 3. The SIMEX estimators were computed with $B=50$. Four hundred data sets were generated and analyzed. The kernel density estimates were calculated as in the previous simulation studies.

Although estimates of the parameters in this model could in theory be analyzed with some 
a. Coefficient of 1

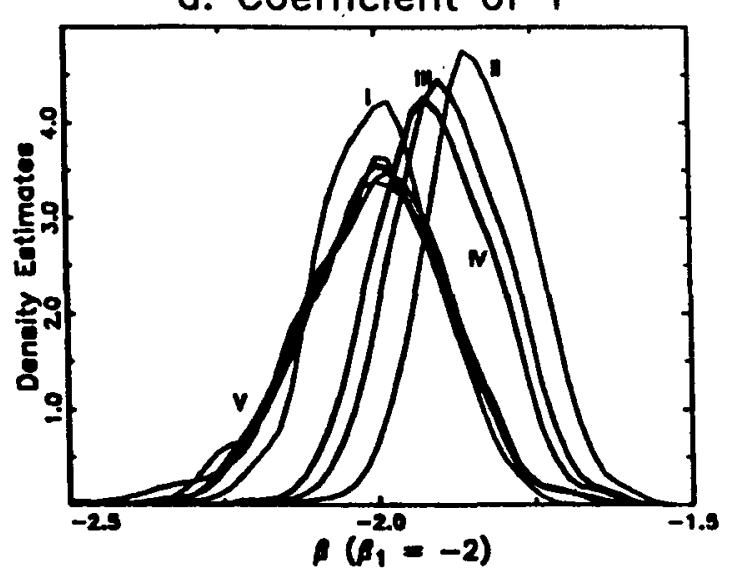

d. Coefficient of 1

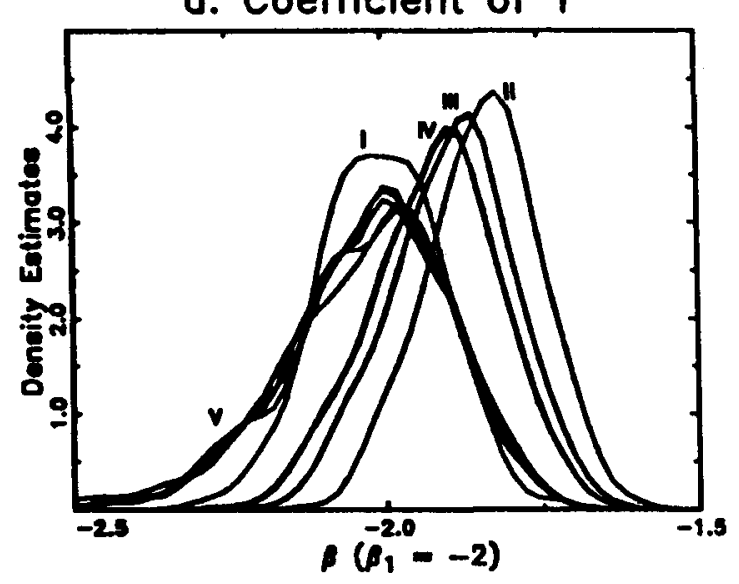

b. Coefficient of $U$

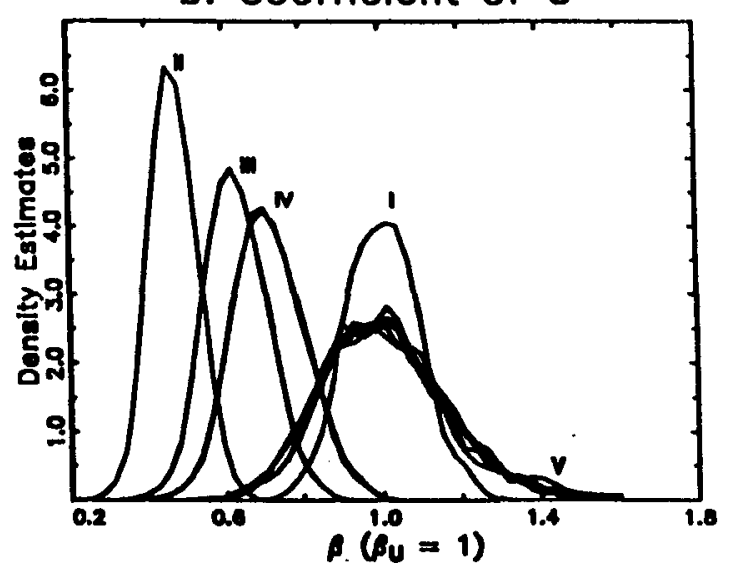

e. Coefficient of $U$

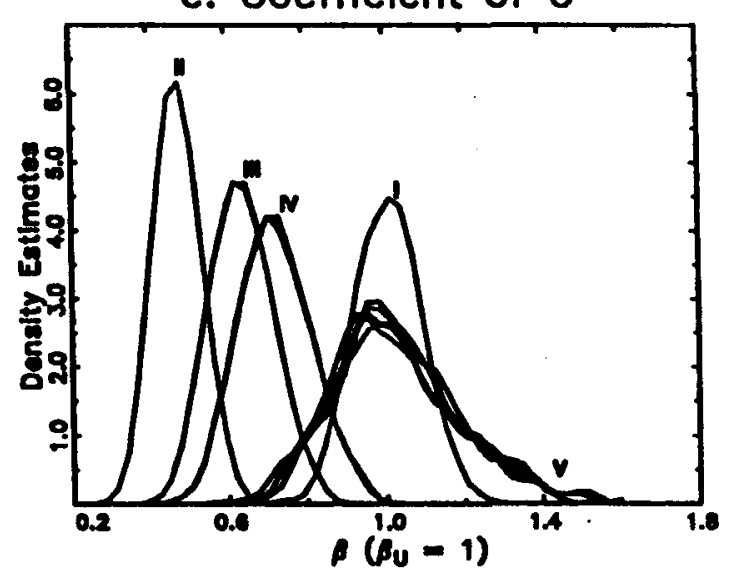

c. Coefficient of $V$

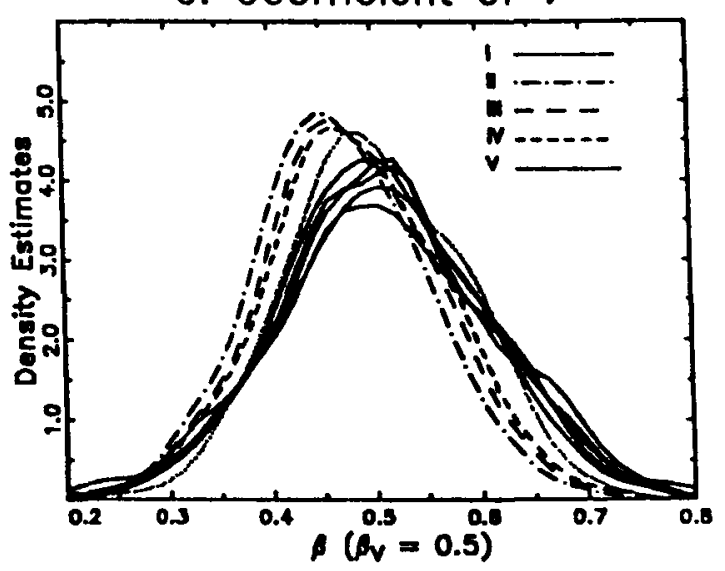

f. Coefficient of $V$

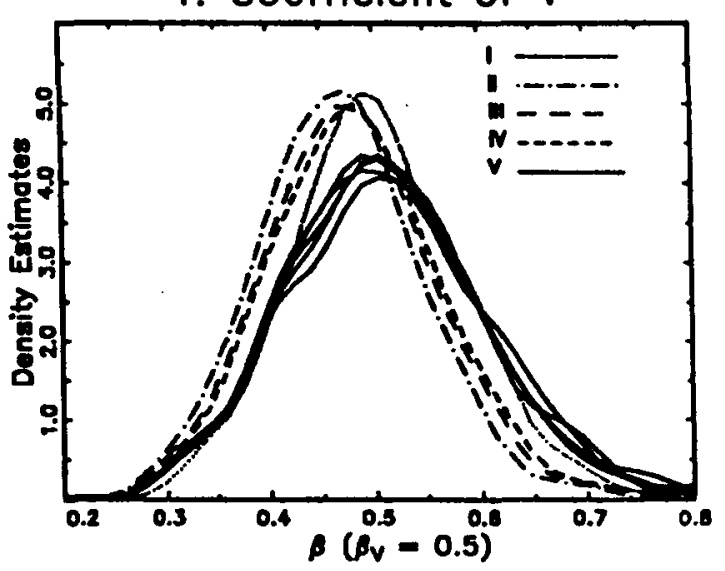

Figures 5a-f. Kernel density estimates of the distributions of the regression parameters in the multiple logistic regression model simulation - ac, normal measurement error; eff, uniform measurement error. 
general approximate estimation procedures (Stefanski, 1985; Fuller, 1987, Ch. 3; Whittemore and Keller, 1988; Carroll and Stefanski, 1990) we are not aware of any results specific to models with interaction terms. Thus there is no benchmark estimator in this study.

The results of the simulation study are displayed in Figures 6a-d. A familiar pattern is apparent. The linear, quadratic and nonlinear variants of the SIMEX estimators all reduce bias with the nonlinear extrapolant yielding a nearly unbiased estimator of all the parameters.

\section{EXPERIENCE with REAL DATA}

The favorable simulation results are very encouraging. However, in applications to real data it is generally the case that all assumptions are violated to some extent, and real data are seldom as amenable to analysis as simulated data. Consequently impressions derived from simulation studies are often optimistic.

The ideal test for our method would require several data sets having both $X$ and $U$ recorded for all observations. Then the SIMEX estimators based on $\left\{Y_{i}, V_{i}, X_{i}\right\}_{1}^{n}$ could be compared to the estimators derived from $\left\{Y_{i}, V_{i}, U_{i}\right\}_{1}^{n}$. This would allow direct assessment of the procedure while blocking for the effects of model misspecification and other violations of assumptions.

Alternatively we might envision a single data set with a large number of replicate measurements, $X_{i, 1}, \ldots, X_{i, m}$, for each observation. The SIMEX estimators based on data including only the $j$ th measurement, $\left\{Y_{i}, V_{i}, X_{i, j}\right\}_{i=1}^{n}$, could be compared with the estimator based on $\left\{Y_{i}, V_{i},, \bar{X}_{i,},\right\}_{1}^{n}$. The latter being a reasonable substitute for the TRUE estimate.

Unfortunately, the two hypothetical experiments just described cannot be realized. However, in the next section we approximate the second experiment by resampling from a data set containing two replicate measurements.

The examples that follow make use of a subset of the data from the Framingham Heart Study. The variables are: $Y$, an indicator of coronary heart disease (CHD) during an eight year period following the second exam; $A$, age at second exam; and $P_{1}$ and $P_{2}$, systolic blood pressure measurements at exams two and three (two and four years post baseline) respectively. Records with missing information were dropped from the data set, leaving a total of 1660 observations. There are 134 cases of CHD. 
a. Coefficient of 1

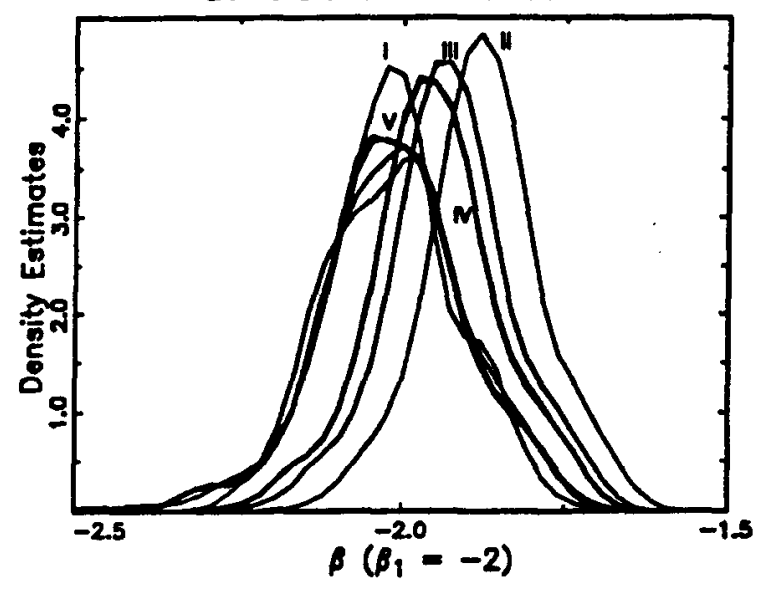

c. Coefficient of $V$

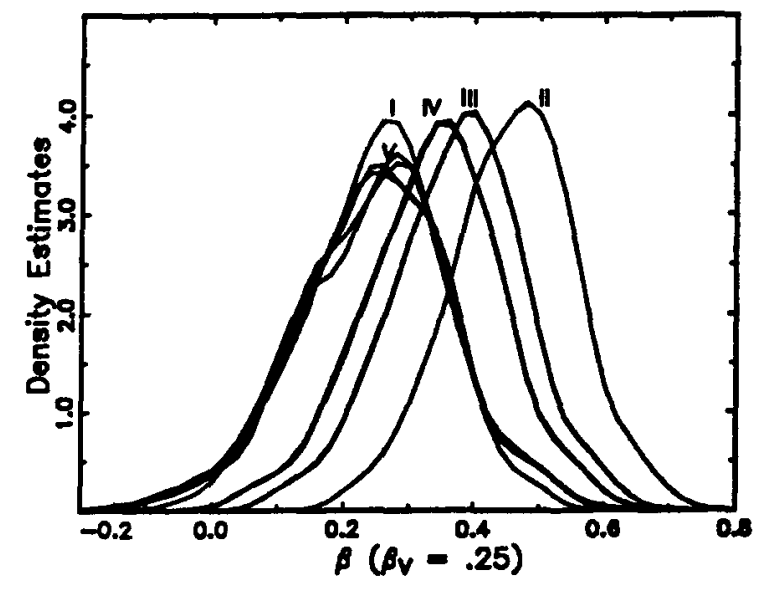

b. Coefficient of $U$

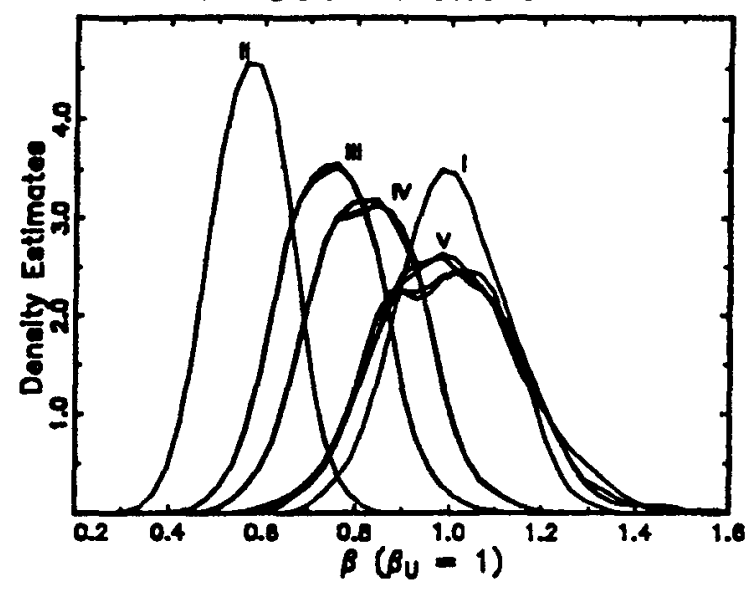

d. Coefficient of UV

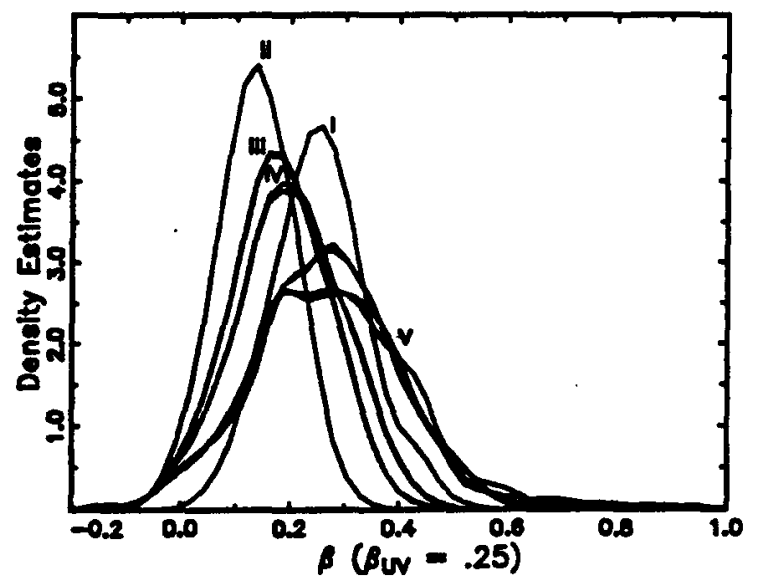

Figures 6a-d. Kernel density estimates of the distributions of the regression parameters in the multiple logistic regression with interaction model simulation. 
We decided to ln-transform systolic pressure and represent age in decades. Thus in what follows $X_{j}=\ln \left(P_{j}\right), j=1,2$, denote the measured predictors, and $V=A / 10$ is a covariate. The true predictor $U$ is thus defined as the natural logarithm of the true SBP, which is defined as a long-term average.

A components-of-variance analysis produced the estimates $\hat{\sigma}_{X}^{2}=.02171$ and $\hat{\sigma}_{U}^{2}=.01563$. Thus the simple linear measurement error model correction for attenuation would be 1.389. This is smaller than the value of 1.6 reported elsewhere (MacMahon et al., 1990), probably because our blood pressure measurements are from exams two years apart whereas MacMahon et al. (1990) use measurements four years apart. The value 1.389 is comparable to the correction for attenuation found by Carroll and Stefanski (1992) using data from the same exams as MacMahon et al. (1990), but employing one measurement as an instrumental variable as a means to account for possible trends over time.

The measurement error variance is estimated to be $\hat{\sigma}^{2}=.006078$, which we assume has negligible sampling variability in the analyses that follow.

\subsection{Blood Pressure and Coronary Heart Disease}

In our first set of analyses we ignore the covariate and consider simple logistic regression modelling of CHD on SBP. Thus we have data $\left\{Y_{i}, X_{i, 1}, X_{i, 2}\right\}_{1}^{1660}$. Let $\bar{X}_{i,}$. denote the average of $X_{i, 1}$ and $X_{i, 2}$. In this study we are going to analyze resampled data sets $\left\{Y_{i}, X_{i}^{*}\right\}_{1}^{1660}$ where $X_{i}^{*}$ is randomly selected to be one of $X_{i, 1}$ or $X_{i, 2}$ with equal probability. With this scheme we create multiple data sets having the same responses and covariates, but with different measured predictors. The variance in a measured predictor is that of a single measurement, $\sigma^{2}=.006078$. One-hundred resampled data sets were generated.

Each resampled data set was analyzed using the nonlinear SIMEX procedure. That is, the naive estimate, $\hat{\beta}_{U}$, and $\left\{\hat{\beta}_{U}\left(\lambda_{j}\right), \lambda_{j}=.5,1,1.5,2\right\}$ were computed and used to fit the nonlinear extrapolant $a+b /(c+\lambda)$. Thus for $b=1, \ldots, 100$ we have the extrapolant curve $\hat{\beta}_{U}^{(b)}(\lambda)$ for $-1 \leq \lambda \leq 2$. Evaluation at $\lambda=0,-1$ yield the naive and nonlinear SIMEX estimators respectively for the $b^{\text {th }}$ generated data set.

Figure $7 a$ displays the pointwise mean extrapolant function $\sum_{b=1}^{100} \hat{\beta}_{U}^{(b)}(\lambda) / 100$ (solid line) and 

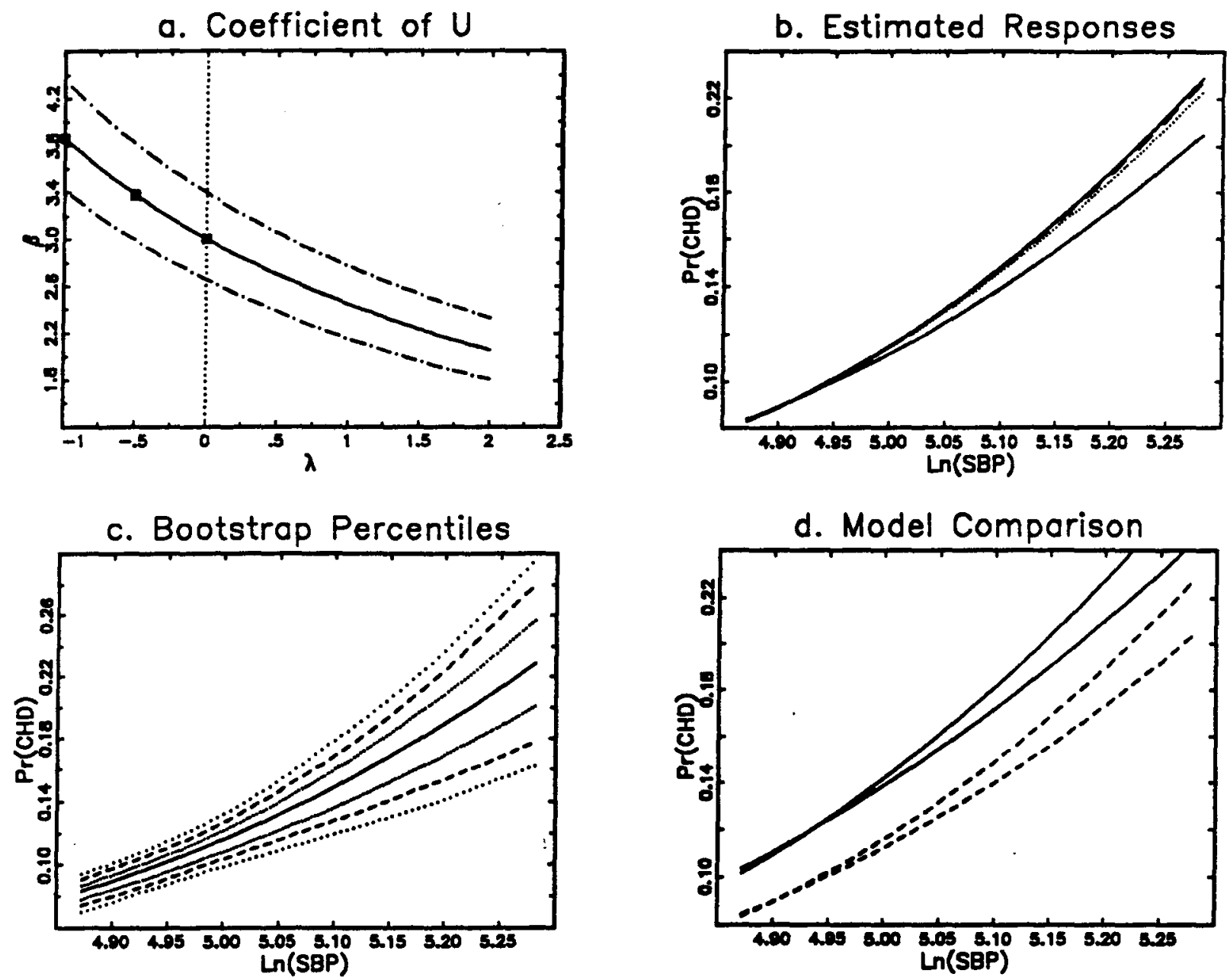

Figure 7a-d. Framingham Data Example. a. Mean extrapolant function with 5th and 95th \%tiles. b. Estimated Response functions for high-risk individuals. Solid, NAIVE and NONLINEAR; dotted, LINEAR; dashed, QUADRATIC. c. Estimated NONLINEAR response function with bootstrap percentiles $(50,7590)$. d. Estimated NAIVE and NONLINEAR response functions for high-risk individuals and models with and without inverse interaction term. Dashed, without interaction; solid, with interaction. 
pointwise fifth and ninety-fifth percentile functions (dotted lines).

The two points plotted at $\lambda=0,-1$ are the means of the naive and nonlinear extrapolant estimators respectively, and lie on the solid curve. The point plotted at $\lambda=-.5$ is the estimate of $\beta_{U}(=3.377)$ obtained by fitting the logistic model to $\left\{Y_{i}, \bar{X}_{i, \cdot}\right\}$ and does not lie on the curve. The fact that it lies so close to the mean extrapolant curve $(=3.383$ at $\lambda=-.5)$ is a consequence of our data generation scheme and the virtual unbiasedness of the nonlinear SIMEX estimator. That is, if our procedure produced exactly unbiased estimates and if an infinite number of resampled data sets had been analyzed, we would expect the curve to pass through the point plotted at $\lambda=-.5$.

To see this, consider that a generated predictor has the representation

$$
X_{i}^{*}=U+\left(\bar{X}_{i, \cdot}-U\right)+\left(T_{i}-.5\right)\left(X_{i, 1}-X_{i, 2}\right)
$$

where $T_{i}$ is a Bernoulli variate with $\operatorname{pr}\left(T_{i}=0\right)=.5=\operatorname{pr}\left(T_{i}=1\right)$. Thus $X_{i}^{*}$ can be written as the sum of $U$ and two uncorrelated errors having zero means and equal variances of $\sigma^{2} / 2$.

The second error can be regarded as the measurement error in $X_{i}^{*}$ as a measure of $\bar{X}_{i, .}$ Thus we would expect the SIMEX procedure to be capable of eliminating the bias due to this error, and return an unbiased estimator of the estimate of $\beta_{U}$ obtained by fitting the logistic model to $\left\{Y_{i}, \bar{X}_{i},\right\}$. Since this error has variance $\sigma^{2} / 2$ and not $\sigma^{2}$, the extrapolant curve should be evaluated at $\lambda=-.5$. In other words, evaluation at $\lambda=-.5$ "corrects" for the bias introduced by the second error, $\left(T_{i}-.5\right)\left(X_{i, 1}-X_{i, 2}\right)$. Evaluation at $\lambda=-1$ corrects for bias caused by the sum of the two errors.

This simulation study demonstrates the near unbiasedness of the procedure with real data. In this example unbiasedness means that the the corrected estimator, $(\lambda=-.5)$, derived from single measurements should, on average, equal the estimate obtained by regressing on the average of of the two measurements. If we had played this game, starting with $m$ measurements instead of two, again generating data sets with one randomly selected measurement, then extrapolation back to $\lambda=-(m-1) / m$ would provide an unbiased estimate of the naive parameter estimate obtained by fitting the model to $\left\{Y_{i}, \bar{X}_{i,}\right\}$.

The simulation study also provides additional evidence of robustness to the assumption of normal errors. The error in $X_{i}^{*}$ as a measure of $\bar{X}_{i}$. has a distribution with only two support points, 
$(t-.5)\left(X_{1}-X_{2}\right), \quad t=0,1$ and is far from normal. Yet the SIMEX procedure, which simulates the effects of measurement error by adding normally distributed errors, proved to be unbiased for this particular case of nonnormal errors.

\subsection{Bootstrapping the SIMEX Procedure}

The simplicity of the SIMEX procedure is offset to some extent by the difficulty of developing asymptotic distribution approximations for the estimators. However, the bootstrap is ideally suited to the task of assessing sampling variability, apart from the obvious computational burden of nested resampling schemes.

In this section we fit the model

$$
\operatorname{pr}(Y=1 \mid U, V)=F\left(\beta_{1}+\beta_{U} U+\beta_{V} V\right)
$$

and use the bootstrap to obtain standard errors and assess the sampling variability in estimated response curves.

Our naive estimate was obtained by fitting (5.1) to $\left\{Y_{i}, \bar{X}_{i . .}, V_{i}\right\}_{1}^{1660}$. The error in $\bar{X}_{i, \text {, as a }}$ measurement of $U_{i}$ is now taken to be $\hat{\sigma}^{2}=.003039(=.006078 / 2)$.

Table 1 displays results from the SIMEX procedure using NON-IID pseudo errors and the sample mean as location functional. Four-hundred bootstrap data sets were analyzed. Table entries are, from top to bottom, point estimates and the means and standard deviations of the 400 bootstrap sample estimates. For comparison we note that the usual inverse-information standard errors of the NAIVE estimates are $2.6594, .56436$ and .10420 for $\beta_{1}, \beta_{U}$ and $\beta_{V}$ respectively.

Figure $7 \mathrm{~b}$ displays estimated response functions for high-risk 50-year old males. The response functions are graphed over the range $\left(\bar{X}_{., .}, \bar{X}_{., .}+3 s\right)$ where $s$ is the standard deviation of $\left\{\bar{X}_{i,}\right\}_{1}^{1660}$.

The two solid curves graph the NAIVE and NONLINEAR SIMEX response curves. The LINEAR and QUADRATIC SIMEX curves are graphed with dotted and dashed lines respectively. The similarity of the SIMEX response functions is due to the small measurement error $\left(\sigma^{2}=.003039\right.$, with a linear correction for attenuation $=1.19)$. For measurement error this small the extrapolant curves are nearly linear and the three methods of extrapolation produce similar results.

Figure 7c displays graphs of the NONLINEAR SIMEX response function (solid line) and selected bootstrap percentiles enclosing central regions of $90 \%$ (dotted), $75 \%$ (dashed) and $50 \%$ (close dots). 


\subsection{Exploratory Data Analysis with Measured Predictors}

Our final example was chosen to emphasize the generality and flexibility of the SIMEX procedure in dealing with nonstandard models. Starting with the naive fit of the model (5.1), we considered the addition of interaction terms of the form $\bar{X}_{i, .}^{r_{1}} V_{i}^{r_{2}}$ for small integer powers $(\{-1,0,1\})$ of $r_{1}$ and $r_{2}$. Taking $r_{1}=r_{2}=-1$ produced the greatest decrease in the deviance, 7.14, although the improvement in the fit is only marginally significant when compared to a Chi-Squared distribution with three degrees of freedom ( $\mathrm{p}$-value $\approx .075$ ). However, the reported p-value is conservative since three is only an approximate degrees of freedom; our model was fit by searching over the two-dimensional finite set of powers, $\left(r_{1}, r_{2}\right)$, and the one-dimensional continuous coefficient space. A model with the measured predictor appearing both on its nominal scale and as an interaction on an inverse scale provides a nice vehicle for illustrating the flexibility of the SIMEX procedure.

Therefore we consider fitting the model

$$
\operatorname{pr}(Y=1 \mid U, V)=F\left(\beta_{1}+\beta_{U} U+\beta_{V} V+\beta_{U V} \frac{1}{U V}\right)
$$

and using the SIMEX procedure to correct for the effects of measurement error.

Table 2 displays coefficient estimates from the SIMEX procedure employing the NON-IID pseudo errors and the sample mean as location estimator. The inclusion of the interaction term induces collinearity, making it difficult to interpret individual coefficients. We present instead a graph similar to that in Figure 7b. Figure 7d displays plots of the NAIVE and NONLINEAR response curves for two models, with and without the inverse interaction term in (5.2). The response functions for the models with and without the interaction term are graphed with solid lines and dotted lines respectively. For both models the SIMEX response function dominates the NAIVE response function over the range plotted.

\section{SUMMARY}

We have shown how the results of several coordinated simulation studies, i.e., the $\{\hat{\theta}(\lambda): \lambda>0\}$, can be used to obtain unbiased or nearly unbiased estimators in nonlinear measurement error models when the measurement error variance is specified. Our experience suggests that employing NONIID pseudo errors and using the sample mean as the location estimator works as well as any other combination of error type and location functional studied, at least in large samples. 
The success of the method is due in large part to the empirically supported general applicability of the nonlinear extrapolant model $a+b /(c+\lambda)$. The beauty of the method is that the appropriate values of the parameters $a, b$ and $c$, which for nonlinear models are no longer simple functions of the data as they are for linear models, are determined by Monte Carlo methods, thereby avoiding mathematical technicalities and/or resorting to approximations of any type.

It seems likely that the basic method can be improved through the use of more creative resampling schemes. We are currently exploring the possibility of combining bootstrap resampling with the Monte Carlo pseudo-error generation in a way that produces both the point estimate and a standard error without the high computational overhead of nested resampling. Also, it may be advantageous to use $\lambda$ values other than $\{.5,1,1.5,2\}$, say by solving an optimal design problem for the mean model $a+b /(c+\lambda)$.

Finally we note that the method is applicable to functional as well as structural measurement error models. This is evident from the results in Sections 4.1, 4.2 and, though not as obviously, from the resampling simulation in Section 5.1. In the latter case, $\bar{X}_{i,}$. remained fixed, as in a functional model. Thus the method does not depend on the nature (random or fixed) of the socalled true values $U_{i}$. This is not true of other methods that explicitly model, either parametrically or non-parametrically, the $U_{i}$ as random variables.

For example, the regression-calibration method (Prentice, 1982; Fuller, 1987, p 261; Rosner et al., 1989,1990; Rudemo et al., 1989; Gleser, 1990; Carroll and Stefanski, 1990; and Pierce et al., 1991) depends on (a model of) $E(U \mid X, V)$. The importance of this is that an estimate of $E(U \mid X, V)$ obtained from external validation/replication data may not "match" the data to be analyzed in the sense that the regressions of $U$ on $(X, V)$ in the validation and target populations need not be equal. However, methods that depend on external validation/replication data only through estimates of $\sigma^{2}$ are generally more robust to differences in the validation and target population distributions. This is obviously true, for example, when measurement error is due entirely to instrument error and the error variance is independently assessed via a designed experiment.

\section{REFERENCES}

Carroll, R. J. \& Stefanski, L. A. (1990) Approximate quasilikelihood estimation in models with 
surrogate predictors. Journal of the American Statistical Association, 85, 652-663.

Carroll, R. J. \& Stefanski, L. A. (1992) Meta-Analysis, Measurement Error and Correction for Attenuation. Statistics in Medicine, to appear.

Cook, J. R. (1989) Estimators for the Errors-in-Variables Problem in the Ordered Categorical Regression Model. Unpublished Ph.D. Thesis, Department of Statistics, North Carolina Stae University.

Fuller, W. A. (1984), “Measurement Error Models With Heterogeneous Error Variances,” in Topics in Applied Statistics eds. Y. P. Chauby and T. D. Dwivedi, Montreal: Concordia University, pp. 257-289.

Fuller, W. A. (1987). Measurement Error Models. Wiley, New York.

Gleser, L. J. (1990). Improvements of the naive approach to estimation in nonlinear errorsin-variables regression models. In Statistical Analysis of Measurement Error Models and Application, P. J. Brown and W. A. Fuller, editors. American Mathematics Society, Providence.

Hwang, J. T. (1986), Multiplicative Errors-In-Variables Models with Applications to the Recent Data Released by U. S. Department of Energy. Journal of the American Statistical Association, $81,680-688$.

MacMahon, S., Peto, R., Cutler, J., Collins, R., Sorlie, P., Neaton, J., Abbott, R., Godwin, J., Dyer, A., \& Stamler, J. (1990). Blood Pressure, Stroke and Coronary Heart Disease: Part 1, Prolonged Differences in Blood Pressure: Prospective Observational Studies Corrected for the Regression Dilution Bias. Lancet, 335, 765-774.

Palca, J. (1990a). Getting to the Heart of the Cholesterol Debate. Science, 24, 1170-71.

Palca, J. (1990b). Why Statistics May Understate the Risk of Heart Disease. Science, $24,1171$.

Pierce, D. A., Stram, D. O., Vaeth, M., Schafer, D. (1991). Some insights into the errors in variables problem provided by consideration of radiation dose-response analyses for the Abomo survivors. Preprint.

Prentice, R. L. (1982). Covariate measurement errors and parameter estimation in a failure time regression model. Biometrika, 69, 331-342.

Rosner, B., Willett, W. C. \& Spiegelman, D. (1989). Correction of logistic regression relative risk estimates and confidence intervals for systematic within-person measurement error. Statistics in Medicine, 8, 1051-1070.

Rosner, B., Spiegelman, D. \& Willett, W. C. (1990). Correction of logistic regression relative risk estimates and confidence intervals for measurement error: the case of multiple covariates measured with error. American Journal of Epidemiology, 132, 734-745.

Rudemo, M., Ruppert, D. \& Streibig, J. C. (1989). Random effect models in nonlinear regression 
with applications to bioassay. Biometrics, 45, 349-362.

Stefanski, L. A. (1985). The effect of measurement error on parameter estimation. Biometrika, 72, 583-592.

Stefanski, L. A. and Carroll, R. J. (1985). Covariate Measurement Error in Logistic Regression. The Annals of Statistics, 13, 1335-1351.

Stefanski, L. A. and Carroll, R. J. (1987). Conditional Scores and Optimal Scores for Generalized Linear Measurement-Error Models. Biometrika, 74, 703-716.

Whittemore, A. S. and Keller, J. B. (1988). Approximations for Regressions with Covariate Measurement Error. Journal of the American Statistical Association, 83, 1057-1066. 
TABLE 1: COMPARISON OF ESTIMATORS. This simulation is described in Section 5.2. Table entries are from top to bottom, point estimates, bootstrap means and bootstrap standard errors. Inverse information standard errors for the NAIVE estimates are 2.6594, .56436 and .10420 for $\beta_{1}, \beta_{U}$ and $\beta_{V}$ respectively.

\begin{tabular}{|c|cccc|}
\hline & NAIVE & LINEAR & QUADRATIC & NONLINEAR \\
\hline$\beta_{1}$ & -17.244 & -18.603 & -18.880 & -19.003 \\
& -17.292 & -18.640 & -18.904 & -19.015 \\
& $(2.5771)$ & $(2.8377)$ & $(2.8821)$ & $(2.8967)$ \\
\hline$\beta_{U}$ & 2.5075 & 2.7998 & 2.8598 & 2.8868 \\
& 2.5144 & 2.8046 & 2.8619 & 2.8862 \\
& $(.54563)$ & $(.60394)$ & $(.61420)$ & $(.61782)$ \\
\hline$\beta_{V}$ & .52814 & .51292 & .50948 & .50771 \\
& .52813 & .51283 & .50943 & .50773 \\
& $(.098739)$ & $(.10028)$ & $(.10068)$ & $(.10092)$ \\
\hline
\end{tabular}

TABLE 2: COMPARISON OF ESTIMATORS. NAIVE and SIMEX estimates for the model with inverse interaction (5.2).

\begin{tabular}{|c|cccc|}
\hline & NAIVE & LINEAR & QUADRATIC & NONLINEAR \\
\hline$\beta_{1}$ & 6.1604 & 5.1932 & 4.9685 & 4.8446 \\
$\beta_{U}$ & 1.0654 & 1.3413 & 1.3974 & 1.4222 \\
$\beta_{V}$ & -1.1709 & -1.2191 & -1.2260 & -1.2278 \\
$\beta_{U V} / 100$ & -1.8645 & -1.8995 & -1.9032 & -1.9038 \\
\hline
\end{tabular}

